\title{
Mucocele frontoetmoidal: relato de casos e revisão da literatura
}

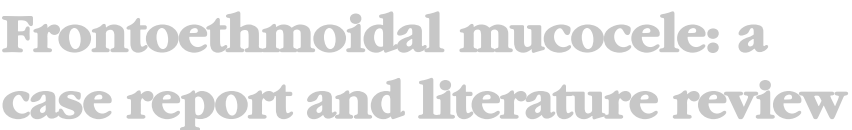

Palavras-chave: mucocele, seios paranasais, cirurgia endoscópica.

Key words: mucocele, paranasal sinus, endoscopic surgery.

\section{Resumo / Summary}

\begin{abstract}
$\mathbf{A}$
mucoceles dos seios paranasais são lesões císticas de revestimento epitelial com conteúdo mucóide, que apresentam crescimento lento com características expansivas e de reabsorção óssea. Eventualmente, podem comprometer as estruturas nobres adjacentes como a órbita e a cavidade intracraniana. Acredita-se que sua etiologia esteja ligada à obstrução do óstio de drenagem do seio paranasal envolvido. Podem causar dor facial, cefaléia, obstrução nasal, diplopia, diminuição da acuidade visual, deslocamento do globo ocular, edema facial ou até mesmo meningite, dependendo da área anatômica comprometida. Acomete mais freqüentemente os seios frontal e etmoidal anterior, sem prevalência de sexo. O diagnóstico é realizado através de exames de imagem, sendo a tomografia computadorizada o exame de eleição, embora em algumas ocasiões a ressonância magnética esteja indicada. Atualmente a abordagem endoscópica paranasal é o acesso cirúrgico de primeira escolha, por ser menos invasiva e apresentar menor morbidade. Este estudo relata dois casos de mucocele frontoetmoidal com extensão orbitária, abordados por via endoscópica endonasal. Os resultados comprovaram a segurança e eficiência deste acesso cirúrgico.
\end{abstract}

\begin{abstract}
Wucoceles of the paranasal sinus are epithelial lesions containing inspissated mucus, slow growth with expansive and osteolytic features that can involve surrounding structures such as orbit and intracranial cavity. The etiology of mucoceles is often associated with ostial obstruction of the compromised sinus. Presenting symptoms can include facial pain, headaches, nasal obstruction, diplopia, decreased visual acuity, orbital globe displacement, facial swelling and meningitis, depending on the anatomic area involved. Mucoceles occur most frequently in the frontal and ethmoidal sinuses, without gender prevalence. The diagnosis is accomplished through imaging. The "gold standard" in terms of diagnostic precision is the computed tomography although the magnetic resonance can be helpful in some cases. Actually transnasal endoscopic management of the mucoceles is preferred due minimal trauma and less morbidity.The present study describes two cases of frontoethmoidal mucoceles with orbital and intracranial involvement that were treated by transnasal endoscopic obtaining good outcomes, demonstrating the security and efficacy of this surgical approach.
\end{abstract}

\footnotetext{
${ }^{1}$ Preceptor da Residência Médica de Otorrinolaringologia do Instituto CEMA. Mestre em Otorrinolaringologia pela UNIFESP/ EPM. ${ }^{2}$ Médicos Residentes de Otorrinolaringologia do Instituto CEMA.

${ }^{3}$ Médico Otorrinolaringologista do Instituto CEMA. Mestrando em Otorrinolaringologia pelo Hospital do Servidor Publico Estadual. ${ }^{4}$ Preceptor da Residência Médica de Otorrinolaringologia do Instituto CEMA. Médico Otorrinolaringologista do HSPM-SP. Coordenador Geral da Residência Médica em Otorrinolaringologia do Instituto Cema. Mestre e Doutor pela UNIFESP/EPM. Endereço para correspondência: Centro de Estudos A/C Sra. Leila. Rua do Oratório 1369 Mooca São Paulo SP 03117-000. Tel (0xx11) 6602.4034 - Fax: 11-6602.4098 - E-mail: centrodeestudos@cemahospital.br Instituto CEMA, São Paulo.

Artigo recebido em 04 de novembro de 2003. Artigo aceito em 11 de dezembro de 2003.
} 


\section{INTRODUÇÃO}

As mucoceles são cistos de conteúdo mucoso que acometem os seios paranasais. Apresentam caráter expansivo, crescimento lento e podem eventualmente comprometer estruturas nobres adjacentes, como a órbita e a cavidade intracraniana ${ }^{1,2,3}$. A marsupialização endoscópica nasossinusal de uma mucocele do seio frontal foi relatada pela primeira vez por Kennedy et al. em 1989. Atualmente, esta via de abordagem cirúrgica é considerada de primeira escolha, por ser menos invasiva e apresentar menor morbidade em relação às técnicas convencionais, como a frontoetmoidectomia externa e os retalhos osteoplásticos com ou sem obliteração do seio frontal ${ }^{1,4-7}$.

O objetivo deste estudo é apresentar dois casos de mucocele frontoetmoidal com extensão orbitária, tratados através de cirurgia endoscópica endonasal e revisão da literatura.

\section{RELATO DE CASOS}

\section{Caso 1}

M.Z.S.M, 20 anos, branca, feminina, com queixa de obstrução nasal intermitente, que piorava em contato com pó e cigarro, associada à cefaléia frontal direita, leve, há cinco meses. Paciente negava outras queixas.

Ao exame físico, presença de abaulamento em canto medial do olho direito, não doloroso à palpação. À rinoscopia anterior foi evidenciado hipertrofia leve de conchas nasais inferiores com palidez de mucosa. O exame endoscópico nasal mostrou discreto abaulamento na região do aggernasi. Foi solicitado tomografia computadorizada de fossas nasais e seios paranasais que demonstrou imagem arredondada, com conteúdo de partes moles na região das células etmoidais anteriores e seio frontal à direita, com deslocamento da lâmina papirácea e globo ocular, além de sinais de remodelação óssea sugerindo quadro compatível com mucocele frontoetmoidal. (Figura 1)

Foi realizado etmoidectomia anterior e sinusotomia frontal endonasal à direita via endoscópica com drenagem e marsupialização da mucocele.

No exame endoscópico pós-operatório ( 2 meses) foi evidenciada a epitelização da cavidade etmoidal, recesso naso-frontal e seio frontal.

\section{Caso 2}

M.A.L, branca, feminina, com queixa de obstrução nasal, cefaléia frontal esquerda com deslocamento do olho ipsilateral, há 6 meses. A paciente nega história prévia de cirurgia nasal ou trauma craniano e não apresentava distúrbios visuais. Ao exame físico, foi observado proptose de olho esquerdo com deslocamento significativo em direção láteroinferior do mesmo. À rinoscopia anterior apresentava abaulamento de meato médio à esquerda. Foi solicitado tomografia computadorizada de fossas nasais e seios paranasais que demonstrou a presença de imagem arredondada, expansiva, com conteúdo de partes moles (tumoração) ocupando fossa nasal, células etmoidais, seio frontal e porção interna da órbita à esquerda, com compressão do globo ocular e músculo reto medial, compatível com mucocele frontoetmoidal.

A ressonância magnética demonstrou processo expansivo frontoetmoidal à esquerda de aspecto benigno sugestivo de mucocele. (Figura 2)

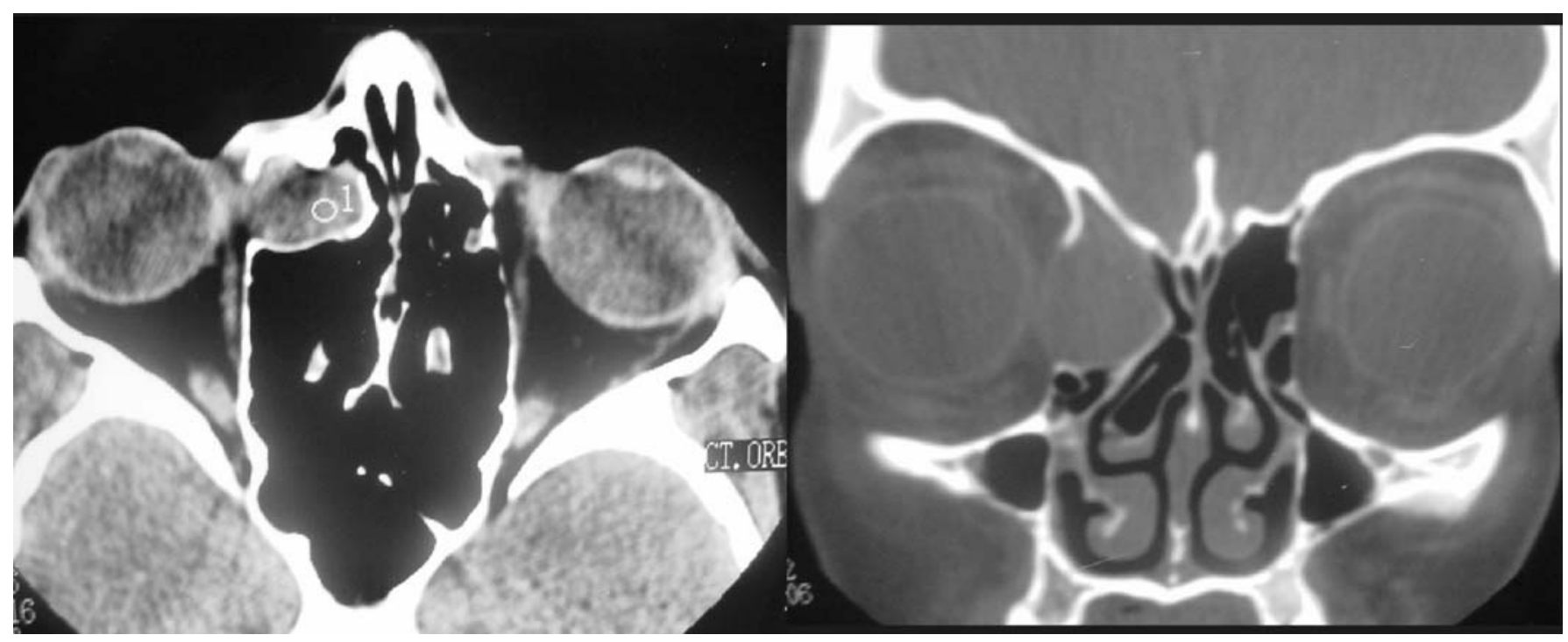

Figura 1. Tomografia Computadorizada de fossas nasais e seios paranasais em cortes axial e coronal evidenciando imagem em região frontoetmoidal direita. 


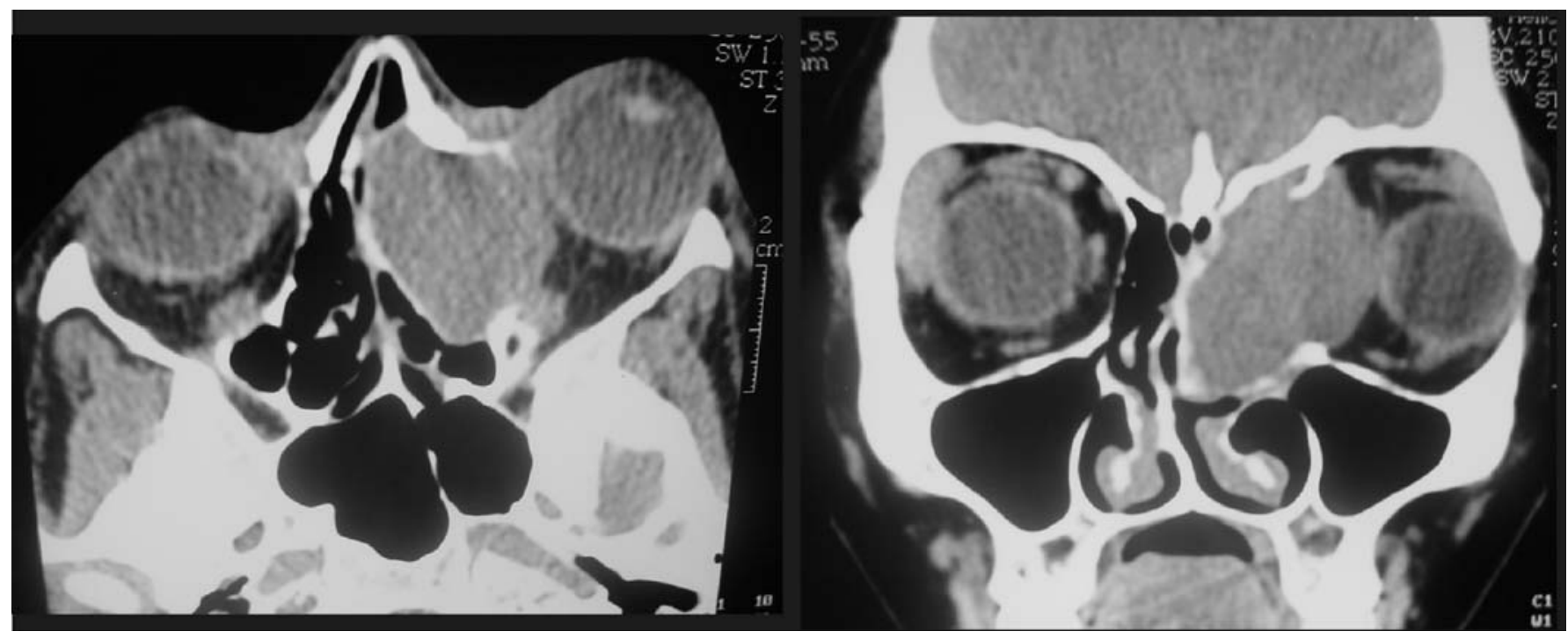

Figura 2. Tomografia Computadorizada de fossas nasais e seios paranasais em cortes axial e coronal evidenciando imagem em região frontoetmoidal esquerda com deslocamento de globo ocular (proptose) esquerda.

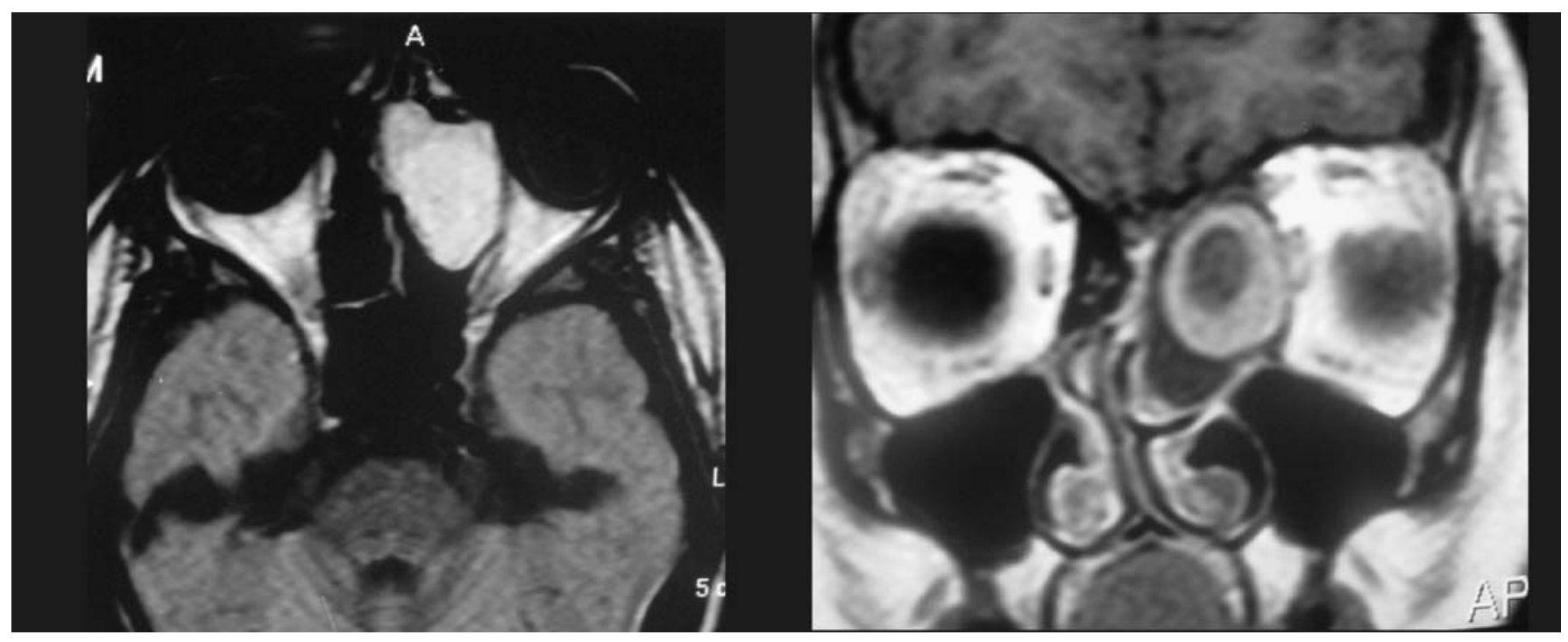

Figura 3. Tomografia Computadorizada de fossas nasais e seios paranasais em cortes axial e coronal com contraste evidenciando imagem em região fronto-etmoidal esquerda com deslocamento de globo ocular (proptose) esquerda.

Foi realizada a drenagem e marsupialização da lesão (com muco espesso no seu interior), sendo evidenciada grande cavidade única, envolvendo os seios etmoidal anterior e frontal com exposição da periórbita à esquerda. A paciente apresentou boa evolução no pós-operatório e o controle com exame endoscópico nasal mostrou cavidade epitelizada, sem sinais de recidiva.

\section{DISCUSSÃO}

Os aspectos clínicos de uma mucocele acometendo o seio frontal foram primariamente descritos por
Langenbeck, em 1819 sendo que Berthon propôs a drenagem cirúrgica para este tipo de afecção em 1880. O termo mucocele foi introduzido por Rollet em 1896 e a primeira descrição histopatológica foi realizada por Onodi em 1901.

A mucocele é uma lesão cística de revestimento epitelial que acomete os seios paranasais, contém muco espesso em seu interior, possui crescimento lento e caráter expansivo $^{1,2}$. Sua etiologia ainda não foi totalmente definida mas acredita-se que seja ocasionada pela obstrução do óstio de drenagem do seio paranasal comprometido devido a processos crônicos de rinossinusite (infecciosa ou alérgica), 
polipose nasossinusal, trauma crânio-facial, cirurgia prévia, tumores benignos (osteomas, displasia fibrosa óssea) ou neoplasias malignas (primárias ou metastáticas) ${ }^{1,3,8-10}$. Ela ocorre mais freqüentemente nos seios frontal e etmoidal, todavia os seios esfenóide e maxilar também podem ser afetados, sendo que neste último a história pregressa de cirurgia de Caldwell-Luc está quase sempre presente ${ }^{3,9,11}$. As mucoceles do seio maxilar são raras, representam menos de $10 \%$ de todas mucoceles de seios paranasais e são mais prevalentes em japoneses ${ }^{11}$. Esta doença tem incidência equivalente entre homens e mulheres e ocorre mais na $3^{\circ} \mathrm{e}$ 4 o décadas de vida.

A mucocele tem propensão de expandir, remodelar e reabsorver as paredes ósseas do seio paranasal acometido, alterando sua integridade e eventualmente comprometendo as estruturas circunvizinhas como a órbita e a cavidade intracraniana ${ }^{2,8}$. A fisiopatologia do mecanismo de reabsorção óssea produzida pela mucocele é obscura. Acredita-se que a osteólise seja produzida pela diminuição da vascularização do osso devido mecanismo de compressão e/ou pela ação de mediadores inflamatórios presentes abundantemente no conteúdo mucoso desta afecção como as citocinas (IL1, IL6), moléculas de adesão vascular, prostaglandinas ${ }^{4,8}$.

O quadro clínico varia conforme a região envolvida, podendo causar dor facial, cefaléia, obstrução nasal, diplopia, diminuição da acuidade visual, deslocamento do globo ocular, edema facial, abscesso cerebral, pneumoencefalocele e meningite ${ }^{1,2,12,13}$. Caso ocorra uma infecção aguda da mucocele, ocasionando uma mucopiocele, é maior a probabilidade de haver uma complicação (orbitária ou intracraniana $)^{10,12}$. Em nosso serviço a queixa dos pacientes envolviam principalmente quadro de obstrução nasal, cefaléia frontal e deslocamento/ abaulamento da órbita com a evolução.

O diagnóstico é feito através dos exames de imagem $^{12}$. Embora o RX simples possa demonstrar opacificação, erosão óssea ou expansão das mucoceles, a tomografia computadorizada (TC) é o exame de eleição, evidenciando o envolvimento ósseo, avaliando a extensão intracraniana e/ou orbitária e auxiliando no planejamento cirúrgico. Na Paciente 1, foi evidenciado na TC imagem arredondada com conteúdo de partes moles ocupando a região de células etmoidais anteriores e seio frontal à direita, deslocando levemente a lâmina papirácea e conseqüentemente o globo ocular, com sinais de remodelação óssea. A Paciente 2 apresentou imagem semelhante à TC. A ressonância magnética (RM) é solicitada quando a (TC) não consegue ser elucidativa, principalmente nos casos que há suspeita de processo neoplásico. A desvantagem da (RM) é sua deficiência em avaliar a anatomia óssea. No caso 2 foi evidenciado processo expansivo frontoetmoidal à esquerda de aspecto benigno.

O diagnóstico diferencial da mucocele inclui as encefaloceles, granuloma de colesterol, cistos epidermóides, meningiomas, cordomas, neurofibromas, adenomas salivares, paragangliomas, nasoangiofibromas ou neoplasias maligna ${ }^{3}$. O tratamento das mucoceles é cirúrgico, sendo que as vias de acesso podem ser externa e endonasal ${ }^{6,7,11,14}$. A abordagem externa é realizada através da frontoetmoidectomia (procedimento de Lynch) ou pelos retalhos osteoplásticos com ou sem obliteração do seio frontal e exanteração total da mucosa ${ }^{7,13}$. Estas técnicas foram por muitos anos as únicas alternativas cirúrgicas para o tratamento das mucoceles frontoetmoidais. São procedimentos agressivos com morbidade elevada e que atualmente são reservados para casos extremos com extensão intracraniana ou orbitária muito significativa ${ }^{1,2}$. A tendência atual é realizar uma abordagem funcional, pouco invasiva e com baixa morbidade, através da cirurgia endoscópica nasossinusal, com marsupialização e drenagem abrangentes da lesão, preservando seu epitélio de revestimento ${ }^{1,2,5,7,10}$. Estudos recentes demonstram que o revestimento da mucocele não perde as características de mucosa respiratória e que com a marsupialização e conseqüente melhora da ventilação local é possível reverter a metaplasia epitelial em epitélio respiratório normal ou pelo menos próximo do normal' . Na Paciente 1 foi realizada etmoidectomia e sinusotomia frontal à direita via endoscópica com drenagem e marsupialização da mucocele, assim como na Paciente 2.

O seguimento a nível ambulatorial dos pacientes com endoscopia nasal de rotina é fundamental para o controle da doença. Os exames de imagem (TC e RM) podem ser úteis, especialmente quando há suspeita de recidiva da lesão.

\section{CONCLUSÃO}

As mucoceles são lesões benignas de caráter expansivo que podem ocasionar sérias complicações a nível orbitário e intracraniano e, por esta razão, devem ser diagnosticadas e tratadas precocemente. A marsupialização com drenagem da lesão via nasossinusal mostrou ser um procedimento seguro e eficiente na abordagem terapêutica das mucoceles frontoetmoidais.

\section{REFERÊNCIAS BIBLIOGRÁFICAS}

1. Chiarini L, Nocini P.F, Bedogni A, Consolo U, Giannetti L, Merli G.A. Intracranial spread of a giant frontal mucocele: case report. British Journal of Oral \& Maxillofacial Sugery 2000; 38: 637-40.

2. Hurley DB, Javer AR, Kuhn FA, Citardi MJ. The endoscopic management of chronic frontal sinusitis associated with frontal sinus posterior table erosion. Am.J.Otolaryngol 2000; 14: 113-20.

3. Lloyd G, Lund VJ, Savy L, Howard D. Radiology in focus. The Journal of Laryngology and Otology 2000; 114: 233-6.

4. Benninger MS, Marks S. The endoscopic management of sphenoid and ethmoid mucoceles with orbital and intranasal extension. Rhinology 1995; 33: 157-61.

5. Har-El G, Dimaio T. Histologic and physiologic studies of marsupialized sinus mucoceles: report of two cases. The Journal of Otolaryngology 2000; 29: 195-8. 
6. Rubin JS, Lund VJ, Salmon B. Frontoethmoidectomy in the treatment of mucoceles. Arch Otolalaryngol Head Neck Surg 1986; 112: 434-6.

7. Ulualp SO, Carlson TK, Toohill RJ. Osteoplastic flap versus modified endoscopic Lothrop procedure in patients with frontal sinus disease. Am J Rhinology 2000; 14: 21-6.

8. Lund VJ, Henderson B, Song Y. Involvement of cytokines and vascular adhesion receptors in the pathology of fronto-ethmoidal mucoceles. Acta Otolaryngol 1998; 113: 540-5.

9. Stiernberg CM, Bailey BJ, Calhoun KH, Quinn FB. Management of invasive frontoethmoidal sinus mucoceles. Arch Otolaryngol Head Neck Surg 1986; 112: 1060-3.
10. Bussaba NY, Salman S.D. Maxillary sinus mucoceles: clinical presentation and long-term results of endoscopic surgical treatment. The Laryngoscope 1999; 109: 1446-9.

11. Busaba NY, Kieff D. Endoscopic sinus surgery for inflammatory maxillary sinus disease. The Laryngoscope 2002; 12: 1378-83.

12. Lund VJ, Rolfe ME. Ophthalmic considerations in fronto-ethmoidal mucoceles. J Laryngol Otol 1989; 103: 667-9.

13. Benninger MS, Steven M. The endoscopic management of sphenoid and ethmoid mucoceles with orbital and intranasal extension. Rhinology 1995; 33: 157-61.

14. Gady H. Endoscopic management of 108 sinus mucoceles. The Laryngoscope 2001; 111: 2131-4. 